\title{
ON LINEAR RECURRENCE SEQUENCES WITH POLYNOMIAL COEFFICIENTS
}

\author{
by A. J. VAN DER POORTEN and I. E. SHPARLINSKI
}

(Received 4 October, 1994)

1. Introduction. We consider sequences $\left(A_{h}\right)$ defined over the field $\mathbb{Q}$ of rational numbers and satisfying a linear homogeneous recurrence relation

$$
S_{0}(h) A_{h+n}=S_{1}(h) A_{h+n-1}+\ldots+S_{n}(h) A_{h}, \quad h=0,1, \ldots,
$$

with polynomial coefficients $S_{j}$. We shall assume without loss of generality, as we may, that the $S_{j}$ are defined over $\mathbb{Z}$ and the initial values $A_{0}, A_{1}, \ldots, A_{n-1}$ are integer numbers.

Also, without loss of generality we may assume that $S_{0}$ and $S_{n}$ have no non-negative integer zero. Indeed, any other case can be reduced to this one by making a shift $h \mapsto h-l-1$ where $l$ is an upper bound for zeros of the corresponding polynomials (and which can be effectively estimated in terms of their heights).

There are a great many familiar sequences satisfying recurrence equations of the present kind (see, say, [6], [11], [17]). Indeed, $\left(A_{h}\right)$ is always the sequence of Taylor coefficients of a formal power series satisfying a linear differential equation with coefficients which are rational functions; a particular case is that of a power series representing an algebraic function (note [17, Theorem 2.1]).

Here we obtain a lower bound for the growth of such sequences. In the special case of recurrence sequences, thus the present sequences with constant coefficients, quite sharp results have been obtained. But even there we do not as yet have an effectively computable exponential lower bound in every case (see [3], [7], [11], [12], [13], [14]).

The height $H(a)$ of a rational number $a=r / s$, where $\operatorname{gcd}(r, s)=1$, is given by $\max (|r|,|s|)$. We write $H_{N}(A)$ for the maximum $\max \left(H\left(A_{0}\right), \ldots, H\left(A_{N-1}\right)\right)$.

Let $\|A\|$ be an upper bound for the height of the initial values $A_{0}, \ldots, A_{n-1}$; that is, $\|A\|=H_{n}(A)$.

It should be well known that if $H_{N}(A)$ is bounded then $\left(A_{h}\right)$ is a periodic sequence; it is therefore a rather special linear recurrence sequence with constant coefficients. The first part of the theorem below gives a quantitative form of this statement (and relies on its qualitative form given in Lemma 1).

Then, in the second part of the theorem we show, by using a modification of the approach of [16], that in the opposite case, that is when $H_{N}(A) \rightarrow \infty$, it is possible to obtain a fairly weak but effectively computable lower bound for $H_{N}(A)$.

We define the length $L(S)$ of a polynomial $S(h) \in \mathbb{Z}[h]$ as the sum of the absolute value of its coefficients. Set

$$
L=\max \left\{\mathscr{L}\left(S_{0}\right), \mathscr{L}\left(S_{1}\right), \ldots, \mathscr{L}\left(S_{n}\right)\right\}, \quad d=\max \left\{\operatorname{deg} S_{0}, \operatorname{deg} S_{1}, \ldots, \operatorname{deg} S_{n}\right\} .
$$

THEOREM. Let $\left(A_{h}\right)$ be a sequence of rationals.

(1) If $H_{N}(A)$ is bounded, then $\left(A_{h}\right)$ is pure periodic with minimal period

and thus

$$
\tau \leq \exp \left((6 n \log n)^{1 / 2}\right) \text {, }
$$

$$
H_{N}(A) \leq\|A\| \exp (O(\tau \log L+d \tau \log \tau)) .
$$

Glasgow Math. J. 38 (1996) 147-155. 
(2) If $H_{N}(A) \rightarrow \infty$ then there exists an effective absolute constant $C>0$ such that

$$
H_{N}(A)>C\left(\frac{\log N}{d \log (L \log N)}\right)^{1 /(2 n+2)} .
$$

These two statements result in an effective test to check whether $H_{N}(A)$ is bounded, and therefore whether $\left(A_{h}\right)$ is periodic. This result is a direct generalization of Theorem 3 of [2] dealing with the corresponding question for sequences satisfying linear recurrence relations with constant coefficients.

2. Bounded $H_{N}(A)$. Here we are dealing with the first case of the theorem. Below we refer to the generating function

$$
f(X)=\sum_{h=0}^{\infty} A_{h} X^{h}
$$

LEMMA 1. Let $\left(A_{h}\right)$ be a sequence of rationals, and suppose that $H_{N}(A)$ is bounded. Then $\left(A_{h}\right)$ is eventually periodic and thus is a sequence satisfying a linear homogeneous recurrence relation with constant coefficients.

Proof. Since inter alia there are only finitely many denominators among the $A_{h}$ we may multiply by their lowest common multiple. Thus we lose no generality in supposing that the $A_{h}$ are integers. Then a theorem of Carlson and Pólya reports (see the comments on Problem 165, Part VIII in Pólya and Szegó [9]; and for more general results see the related discussion of Amice [1, Chapter 5]) that the generating function $f(X)$ is either rational or has the unit circle as a natural boundary.

On the other hand it is well known (see for example [5] but note that this result can also be found in [17]), that the generating function satisfies a linear differential equation with polynomial coefficients (Lemma 2 below gives a slightly more precise form of this statement). Functions satisfying a linear differential equation with polynomial coefficients do not have a natural boundary. Hence if $H_{N}(A)$ is bounded then $f(X)$ must represent a rational function.

So the sequence $\left(A_{h}\right)$ satisfies a linear homogeneous recurrence relation with constant coefficients and so, from some $h$ on, the $A_{h}$ are given by a generalised power sum

$$
A_{h}=\sum_{i=1}^{m} P_{i}(h) \alpha_{i}^{h}
$$

Since the $A_{h}$ are integers and are bounded it follows that the roots $\alpha_{i}$ must be roots of unity and that the polynomial coefficients $P_{i}(h)$ must be constants. Hence the sequence $\left(A_{h}\right)$ is, as asserted, eventually periodic.

Lemma 2. The function $f(X)$ satisfies a differential equation

$$
\sum_{i=0}^{d} R_{i}(X) f^{(i)}(X)=R_{-1}(X),
$$

where the $R_{i}(X), i=-1,0, \ldots, d$, are polynomials with coefficients not all equal to zero and of degree at most $n$. 
Proof. Having defined the differential operators

one sees that

$$
D_{i}=\frac{d^{i}}{i ! d X^{i}}, \quad i=0,1, \ldots,
$$

$$
D_{i} f(X)=\sum_{h=0}^{\infty} A_{h}\left(\begin{array}{l}
h \\
i
\end{array}\right) X^{h-i}, \quad i=0,1, \ldots
$$

(under the standard agreement that $\left(\begin{array}{c}h \\ i\end{array}\right)=0$ for $h<i$ ). Further, we define the coefficients $t_{i, j}$ by the representations

$$
T_{j}(X)=\sum_{i=0}^{d} t_{i, j}\left(\begin{array}{l}
h \\
i
\end{array}\right) X^{i}, \quad j=0, \ldots, n,
$$

where $T_{n}(X)=S_{n}(X)$ and $T_{j}(X)=-S_{j}(X), j=1, \ldots, n-1$. Therefore, from (1) we obtain

$$
\begin{aligned}
\sum_{i=0}^{n} \sum_{i=0}^{d} t_{i, j} X^{i} D_{i} \sum_{h=0}^{\infty} A_{h+j} X^{h} & =\sum_{j=0}^{n} \sum_{i=0}^{d} t_{i, j} \sum_{h=0}^{\infty} A_{h+j}\left(\begin{array}{l}
h \\
i
\end{array}\right) X^{h+j} \\
& =\sum_{h=0}^{\infty} X^{h} \sum_{j=0}^{n} A_{h+j} \sum_{i=0}^{d} t_{i, j}\left(\begin{array}{l}
h \\
i
\end{array}\right) \\
& =\sum_{h=0}^{\infty} X^{h} \sum_{j=0}^{n} A_{h+j} T_{j}(h)=0 .
\end{aligned}
$$

Multiplying the last identity by $n ! X^{n}$ and changing the order of the summation, we get

where

$$
\sum_{i=0}^{d} R_{i}(X) f^{(i)}(X)=R_{-1}(X)
$$

and

$$
R_{i}(X)=\frac{n !}{i !} \sum_{j=0}^{n} t_{i, j} X^{n+i-j}, \quad i=0, \ldots, d,
$$

$$
R_{-1}(X)=\sum_{i=0}^{d} \frac{n !}{i !} \sum_{j=0}^{n} t_{i, j} X^{n+i-j} \sum_{h=0}^{j-1} A_{h}
$$

It is evident that at least one of the polynomials above is not identical to zero.

Proof of the theorem: Part 1. As in the proof of Lemma 1 we obtain a representation (2) of the $A_{h}$ as a power sum with the $P_{i}(h)$ constants and the $\alpha_{i}$ roots of unity, $i=1, \ldots, m$. Thus the only poles of the generating function $f(X)$ are the reciprocals $1 / \alpha_{i}$, $i=1, \ldots, m$, and they are roots of unity. On the other hand, it follows from Lemma 2 that all poles of $f(X)$ are zeros of the first non-zero polynomial among $R_{d}, R_{d-1}, \ldots, R_{0}$. Each is of degree at most $n$. Therefore, the degree of the field extension $\mathbb{Q}\left(\alpha_{1}, \ldots, \alpha_{m}\right)$, is at most $n$. Also, it is well known that if $\alpha$ is a root of unity of degree $N$ and $T$ is the minimal positive integer $t$ such that $\alpha^{t}=1$-we shall refer to $T$ as the period of $\alpha$-then $\phi(T)=N$, where $\phi(T)$ is the Euler function. Thus if $T_{1}, \ldots, T_{m}$ are the periods of $\alpha_{1}, \ldots, \alpha_{m}$, respectively, then $\tau \leq \operatorname{LCM}\left(T_{1}, \ldots, T_{m}\right)$. The maximal possible value of this 
least common multiple is estimated in $[2$, Theorem 1$]$ as $\exp \left((6 n \log n)^{1 / 2}\right)$ and the bound for $\tau$ follows.

Moreover, $\left(A_{h}\right)$ is pure periodic. Assume that this is not so, and that $k$ is minimal so that $A_{k} \neq A_{k-\tau}$. Then $A_{k+r}=A_{k+r-\tau}$ for all integer $r \geq 1$. In particular, from (1) we obtain

$$
\begin{aligned}
S_{0}(k+l \tau) A_{k+n}- & S_{1}(k+l \tau) A_{k+n-1}-\ldots-S_{n}(k+l \tau) A_{k} \\
& =S_{0}(k+l \tau) A_{k+l \tau+n}-S_{1}(k+l \tau) A_{k+l \tau+n-1}-\ldots-S_{n}(k+l \tau) A_{k+l \tau}=0
\end{aligned}
$$

for all positive integers $l$. Therefore we have the polynomial identity

It yields

$$
S_{0}(X) A_{k+n}-S_{1}(X) A_{k+n-1}-\ldots-S_{n}(X) A_{k}=0 .
$$

$$
\begin{aligned}
S_{n}(k-\tau) A_{k-\tau} & =S_{0}(k-\tau) A_{k+n-\tau}-S_{1}(k-\tau) A_{k+n-1}-\ldots-S_{n-1}(k-\tau) A_{k-\tau+1} \\
& =S_{0}(k-\tau) A_{k+n}-S_{1}(k-\tau) A_{k+n-1}-\ldots-S_{n-1}(k-\tau) A_{k+1} \\
& =S_{n}(k-\tau) A_{k} .
\end{aligned}
$$

As the polynomial $S_{n}(h)$ has no integer positive zeros, we obtain $A_{k-\tau}=A_{k}$, and this contradiction shows that $A_{h}=A_{h+\tau}$ for all $h=0,1, \ldots$

Finally, we notice that from (1) we have the representation

$$
A_{h}=\frac{Q_{h}}{\prod_{k=0}^{h-n} S_{0}(k)}, \quad h=0,1, \ldots,
$$

where $Q_{h}$ are integers satisfying the recurrence equation

$$
Q_{h+n}=\sum_{i=1}^{n} S_{i}(h) Q_{h+n-i} \prod_{k=h-i+1}^{h} S_{0}(k), \quad h=0,1, \ldots
$$

Therefore,

$$
\left|Q_{h+n}\right| \leq \sum_{i=1}^{n}\left(L(h+1)^{d}\right)^{i+1}\left|Q_{h+n-i}\right|, \quad h=0,1, \ldots
$$

Together with

$$
\max \left\{\left|Q_{0}\right|, \ldots,\left|Q_{n-1}\right|\right\}=\max \left\{\left|A_{0}\right|, \ldots,\left|A_{n-1}\right|\right\}=\|A\|
$$

we obtain the estimate $\left|Q_{h+n}\right| \leq 2\|A\|\left(L(h+1)^{d}\right)^{2 h}$. Also, of course we have

$$
\left|\prod_{k=0}^{h} S_{0}(k)\right| \leq \prod_{k=0}^{h} L(k+1)^{d}=L^{h+1}((h+1) !)^{d} .
$$

Therefore $H_{h}(A) \leq H_{h+n}(A)=\|A\| \exp (O(h \log L+d h \log h))$ for any $h$. On replacing $h$ by $\tau$ we obtain the asserted bound for $H_{N}(A)$.

3. Unbounded $H_{N}(A)$. Here we consider Part 2 of the theorem: $H_{N}(A) \rightarrow \infty$. The proof relies on Lemmata 3-5 but first of all we recall some standard notation and several well known facts. 
For a polynomial $S(h) \in \mathbb{Z}[h]$, beside its length $\mathscr{L}(S)$, we define its height $H(S)$ simply as the maximum absolute value of its coefficients, and its Mahler measure as

$$
\mathcal{M}(S)=s_{0} \prod_{j=1}^{\text {deg } S} \max \left\{1,\left|\sigma_{j}\right|\right\},
$$

where $s_{0}$ is the leading coefficient of $S$ and the product is over all zeros of $S$ repeated according to their multiplicity.

Then the following inequalities can be found in [8]:

$$
H(S) \leq \mathscr{L}(S) \leq(\operatorname{deg} S+1) H(S), \quad \mathcal{M}(S) \leq \mathscr{L}(S) \leq 2^{\operatorname{deg} S} \mathcal{M}(S) .
$$

For $S(h), T(h) \in \mathbb{Z}[h]$, it is evident that $\mathcal{M}(S T)=\mathcal{M}(S) \mathcal{M}(T)$, whence the previous inequalities yield

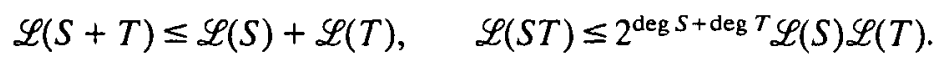

LEMMA 3. For every nonnegative integer l there are polynomials

$$
S_{i, l}[h] \in \mathbb{Z}[h], \quad i=1, \ldots, n,
$$

of degree at most ld and of length at most

such that

$$
L_{l}=n^{l} 2^{n l(l+1) / 2} L^{n l}
$$

$$
S_{0}^{\prime}(h) A_{h+l}=S_{1, l}(h) A_{h+n-1}+\ldots+S_{n, l}(h) A_{h}, \quad h=1,2, \ldots
$$

Proof. We use induction on $l$. For $l \leq n-1$ the assertion is trivial and for $l=n$ we may set $S_{i, n}=S_{i} S_{0}^{n-1}, i=1, \ldots, n$. It is clear that $\operatorname{deg} S_{i, n} \leq n d$ and that

$$
\mathscr{L}\left(S_{i, n}\right) \leq 2^{\text {nd }} \mathcal{M}\left(S_{i, n}\right) \leq 2^{n d} L^{n} \leq L_{n}, \quad i=1, \ldots, n .
$$

For $l>n$ we have

$$
\begin{aligned}
S_{0}^{\prime}(h) A_{h+l} & =\sum_{j=1}^{n} S_{j}(h) S_{0}(h)^{\prime-1} A_{h+l-j}=\sum_{j=1}^{n} S_{j}(h) S_{0}(h)^{j-1} \sum_{i=1}^{n} S_{i, l-j}(h) A_{h+n-i} \\
& =\sum_{i=1}^{n} A_{h+n-i} \sum_{j=1}^{n} S_{j}(h) S_{0}(h)^{j-1} S_{i, l-j}(h),
\end{aligned}
$$

and we may set

$$
S_{i, l}=\sum_{j=1}^{n} S_{j} S_{0}^{j-1} S_{i, l-j} .
$$

But each term $S_{j} S_{0}^{j-1} S_{i, l-j}$ in the last sum is a polynomial of degree at most $l d$ and of length at most

Hence

$$
\begin{aligned}
\mathscr{L}\left(S_{j} S_{0}^{j-1} S_{i, l-j}\right) & \leq 2^{l d} \mathcal{M}\left(S_{j} S_{0}^{j-1} S_{i, l-j}\right) \\
& =2^{l d} \mathcal{M}\left(S_{j}\right) \mathcal{M}\left(S_{0}\right)^{j-1} \mathcal{M}\left(S_{i, l-j}\right) \leq 2^{l d} L^{n} L_{l-j} \leq 2^{l d} L^{n} L_{l-1} .
\end{aligned}
$$

$$
L_{l} \leq n 2^{l d} L^{n} L_{l-1} \leq n^{l} 2^{d l(l+1) / 2} L^{n l},
$$

and we are done. 
It will be convenient to use a compact notation whereby we write

$$
\mathfrak{Q}_{h}^{j}=A_{h}^{j_{0}} \ldots A_{h+n-1}^{j_{n-1}}
$$

for a monomial of total degree $|j|=j_{0}+\ldots j_{n-1}$ in $n$ consecutive $A$ 's.

LEMMA 4. For each pair of integers $l \geq 0$ and $k=|j|$ there is a polynomial

$$
G_{j, l}[h] \in \mathbb{Z}[h], \quad j=\left(j_{0}, \ldots, j_{n-1}\right)
$$

of degree at most kld and of length at most

such that

$$
\Lambda_{l, k}=n^{k(l+1)} 2^{d k l(l+3) / 2} L^{k n l}
$$

$$
S_{0}^{k l}(h) A_{h+1}^{k}=\sum_{\mid j=k} G_{j, l}(h) \mathfrak{Q}_{h}^{j} .
$$

Proof. It follows from Lemma 3 that we may set

where

$$
G_{j, l}=C(j) \prod_{i=0}^{n-1} S_{n-i, l}^{j_{i}}
$$

$$
C(j)=\frac{k !}{j_{1} ! \ldots j_{n-1} !} \leq n^{k}
$$

Therefore $\operatorname{deg} G_{j, l} \leq k l d$ and

$$
\mathscr{L}\left(G_{j, l}\right) \leq n^{k} 2^{k l d} M\left(\prod_{i=0}^{n-1} S_{n-i, l}^{j_{i}}\right) \leq n^{k} 2^{k l d} L_{l}^{k}=n^{k(l+1)} 2^{d k l(l+3) / 2} L^{k n l} .
$$

LEMMA 5. A linear homogeneous system of $M$ polynomial equations

$$
\sum_{l=0}^{M} P_{j, l}(h) Q_{l}(h)=0, \quad j=1, \ldots, M
$$

with $M+1$ unknowns $Q_{l}$, polynomials $P_{j, l} \in \mathbb{Z}[h], l=0, \ldots, M$ and $j=1, \ldots, M$, of degree at most $\delta$ and height at most $H$, has a nonzero solution in polynomials of degree at most $\Delta=\delta M$ and of height at most $((M+1) H)^{M+1}$.

Proof. The data amounts to our having a linear homogeneous system of

$$
(\delta+\Delta+1) M<(\Delta+1)(M+1)
$$

equations with coefficients whose absolute values do not exceed $H$, in the

$$
(\Delta+1)(M+1)
$$

unknown integer coefficients of the polynomials $Q_{l}$ of degree at most $\Delta$. By "Siegel's lemma" such a system has a nonzero integer solution of height not exceeding $((M+1) H)^{M+1}$ (see [4] for a survey of various sharpenings of this result).

Proof of the theorem: Part 2. Set $H_{N}(A)=Q$ and consider the polynomial

$$
\Psi(\alpha)=\prod_{u=1}^{Q} \prod_{v=-Q}^{Q}(u \alpha-v)
$$


of degree $D=(2 Q+1) Q$, and height $H(\Psi) \leq 2^{D} Q^{D}<D^{D}$. Define the sequence $\left(W_{h}\right)$ by $W_{h}=\Psi\left(A_{h}\right)$. If $\Psi(h)=\sum_{k=0}^{D} B_{k} h^{k}$ then, by Lemma 4 we have the representation

$$
S_{0}^{D l}(h) W_{h+l}=\sum_{k=0}^{D} B_{k} S_{0}^{(D-k) l}(h) \sum_{|j|=k} G_{j, l}(h) \mathfrak{U}_{h}^{j}
$$

Hence we may write, for non-negative integer $l$,

$$
S_{0}^{D l}(h) W_{h+l}=\sum_{|j| \leq D} P_{j, l}(h) \mathbb{P}_{h}^{j}, \quad h=1,2, \ldots,
$$

with polynomials $P_{j, l}$ of degree at most $l d D$ and of height at most

$$
\begin{aligned}
H_{l}(D) & =\max _{k=0, \ldots, D}\left|B_{k}\right| 2^{(D-k) l d} L^{D-k} \Lambda_{l, k} \\
& =\max _{k=0 \ldots, D}\left|B_{k}\right| 2^{(D-k) l d} L^{D-k} n^{k(l+1)} 2^{d k l(l+3) / 2} L^{k n l} \\
& \leq H(\Psi) n^{D(l+1)} 2^{d D l(l+3) / 2} L^{D n l} \leq \exp \left(c_{1} d n \log L D \log D\right) ;
\end{aligned}
$$

here $c_{1}>0$ is some absolute constant.

The number of $j=\left(j_{0}, \ldots, j_{n-1}\right)$ with $|j| \leq D$ is $M=\left(\begin{array}{c}D+n \\ n\end{array}\right)$. Accordingly, we consider the system of $M$ equations

$$
\sum_{l=0}^{M} P_{j, l}(h) Q_{l}(h)=0, \quad j=1, \ldots, M,
$$

in the $M+1$ unknowns $Q_{l}(h), l=0, \ldots, M$. By (3) we have that

$$
\sum_{l=0}^{M} S_{0}^{D l}(h) Q_{l}(h) W_{h+l}=0, \quad h=1,2, \ldots
$$

From the bounds for $H_{l}(D)$ and Lemma 5 we see that for some $m \leq M$ and some polynomials $R_{l} \in \mathbb{Z}[h], l=0, \ldots, m$, the sequence $\left(W_{h}\right)$ satisfies a linear recurrence equation

$$
\sum_{l=0}^{m} R_{l}(h) W_{h+l}=0, \quad h=1,2, \ldots,
$$

of order $m$ and with polynomial coefficients of height

$$
H \leq \exp \left(c_{2} d n \log L D^{n+1} \log D\right),
$$

where $c_{2}>0$ is some absolute constant.

Clearly, $W_{h}=0$ for $h=0, \ldots, N-1$. On the other hand, since $H_{N}(A) \rightarrow \infty$ the sequence has nonzero values. But any integer zero of the polynomial $R_{m}(h)$ is plainly at most $H$ (because it divides the constant coefficient of $R_{m}$ ). Hence, of $m$ consecutive 
values $W_{h}, h=H+1, \ldots, H+m$, at least one must be nonzero. Thus $H+m \geq N$. The upper bounds for $H$ and $m$ now yield the result.

4. Several remarks. As mentioned above, the theorem leads to an effective test to check whether the sequence $\left(A_{h}\right)$ is periodic. It is enough to compute $H_{N}(A)$ for some $N$ for which the estimates of the first and the second parts of the theorem contradict one another.

Unfortunately, such an $N$ is astronomically large, being multi-exponential with respect to all its parameters. Indeed, suppose we define the "input-size" as $I=$ $n \log \|A\|+n d \log L+1$. Then the minimal $N$ up to which we have to compute $H_{N}(A)$ is of order

$$
N(I)=\exp \exp \exp \left(I^{1 / 2+\varepsilon}\right)
$$

For $n$ fixed this can be reduced to $N(I)=\exp \exp \left(I^{1+\varepsilon}\right)$. A natural question is if one can do better. In particular we would like to stress the importance of the following problem.

OPEN QUeSTION 1. Is it possible to determine whether $H_{N}(A) \rightarrow \infty$ in polynomial time $T=I^{O(1)}$ ?

For linear recurrence sequences over an arbitrary field $\mathbb{K}$ it is natural to consider the number $V_{N}(A)$ of different values among $A_{1}, \ldots, A_{N}$, instead of the height $H_{N}(A)$.

It is not difficult to see that the method used here allows one to prove that the sum and product of any two linear recurrence sequences satisfying equations of the shape (1) over an arbitrary field $\mathbb{K}$ are linear recurrence sequences of the same type with explicit bounds for the order of their corresponding equations and the degree of their coefficients. This is an explicit version of the well known fact that the set of all linear recurrence sequences satisfying equations of the shape (1) over an arbitrary field $\mathbb{K}$ form a ring ([6], [11], [17]).

For example, in [15], the aforementioned result of [2] was generalized to linear recurrence sequences of algebraic numbers. Accordingly our results can be extended to this case as well.

Unfortunately, over an arbitrary field we cannot readily bound the maximal zero of a polynomial; yet that is a crucial point of the final part of our method.

On the other hand, if all the coefficients of the equation (1) over an arbitrary field $\mathbb{K}$ are constant, then one can readily obtain

$$
V_{N}(A) \geq N^{1 / n}
$$

for the number $V_{N}(A)$ of different values among $A_{1}, \ldots, A_{N}$. This holds provided that $N \leq \tau$ where $\tau$ is a period of the linear recurrence sequence, if such exists (for example in the case of a finite field $\mathbb{K}=\mathbb{F}_{q}$ ); otherwise we formally set $\tau=\infty$. Indeed, if $N \leq \tau$ then all $N$ vectors $\left(A_{h}, \ldots, A_{h+n-1}\right), h=1, \ldots, N$, must be distinct, therefore $V(N)^{n} \geq N$. There are improvements on this idea in [16] in the case of a finite field $\mathbb{K}=\mathbb{F}_{q}$.

In respect of the general case we ask:

Open Question 2. Does the collection of all linear recurrence sequences, satisfying equations with leading coefficient $S_{0}(h)$ without an integer zero, form a ring?

Given a positive answer to this question, one can bound $V_{N}(A)$ from below in any 
field, independent of any bounds for the height of the polynomials coefficients or for their zeros. Certainly a most interesting generalization would be on sequences over $p$-adic fields. The following question could be the first step in this direction.

OPEN QUESTION 3. Can the approach of this paper be adjusted to get non-trivial lower bounds in p-adic norms of sequences of rational numbers satisfying the equation (1)?

ACKNowledgment. The authors would like to thank the referee for many valuable comments, in particular, for the question about the possibility of a $p$-adic generalization.

This work was supported in part by grants from the Australian Research Council and a research agreement with Digital Equipment Corporation.

\section{REFERENCES}

1. Y. Amice, Les nombres p-adiques (Presses Universitaires de France, 1975).

2. J. Berstel and M. Mignotte, Deux properties decidables des suites recurrences linearies, Bull. Soc. Math. France 104 (1976), 175-184. $225-244$.

3. J.-H. Evertse, On sums of $S$-units and linear recurrences, Compositio Math. 53 (1984),

4. M. R. Flahive, Integral solutions of linear systems, in Number theory, J.-M. De Koninck, ed. (Walter de Gruyter \& Co., Berlin, 1989), 213-219.

5. L. Lipshitz, $D$-finite power series, J. Algebra 122 (1989), 353-373.

6. L. Lipshitz and A. J. van der Poorten, Rational functions, diagonals, automata and arithmetic, in Number theory, R. A. Mollin, ed., (Walter de Gruyter \& Co., Berlin, 1990), 339-358.

7. J. H. Loxton and A. J. van der Poorten, On the growth of recurrence sequences, Math. Proc. Camb. Phil. Soc. 81 (1977), 369-376.

8. M. Mignotte, An inequality about irreducible factors of integer polynomials, J. Number Theory 30 (1988), 156-166. 1975).

9. G. Pólya and G. Szegô, Problems and theorems in analysis, 4th edition, (Springer-Verlag,

10. A. J. van der Poorten, Some facts that should be better known, especially about rational functions, in Number theory and applications, R. A. Mollin ed., (Kluwer Acad. Publ., The Netherlands, 1989), 497-528.

11. A. J. van der Poorten, Power series representing algebraic functions, in Séminaire de théorie des nombres, Paris 1990-91, S. David, ed. (Birkhäuser, Boston, 1992), 241-262.

12. A. J. van der Poorten and H. P. Schlickewei, Zeros of recurrence sequences, Bull. Austral. Math. Soc. 44 (1991), 215-223.

13. A. J. van der Poorten and H. P. Schlickewei, Additive relations in fields, J. Austral. Math. Soc. 51 (1991), 154-170.

14. A. J. van der Poorten and I. E. Shparlinski, On the number of zeros of exponential polynomials and related questions, Bull. Austral. Math. Soc. 46 (1992), 401-412.

15. P. Robba, Zeros de suites recurrentes linearies, Groupe d'etude d'Analyse ultrametrique, 13 (1977/78) 1-5.

16. I. E. Shparlinski, On the distribution of values of recurring sequences and the Bell numbers in finite fields, Europ. J. Combinatorics, 12 (1991), 81-87.

17. R. P. Stanley, Differentiably finite power series, Europ. J. Combinatorics, 1 (1980), $175-188$.

Centre for Number Theory Research

School of Mathematics, Physics, Computing and Electronics

MACQUARIE UNIVERSITY NSW 2109

Australia 